\title{
Severity of imported malaria: protective effect of taking malaria chemoprophylaxis
}

\author{
Klaske Vliegenthart-Jongbloed ${ }^{1}$, Mariana de Mendonça Melo ${ }^{1}$, Marlies E van Wolfswinkel ${ }^{1}$, Rob Koelewijn², \\ Jaap J van Hellemond ${ }^{2,3}$ and Perry JJ van Genderen ${ }^{\text {l* }^{*}}$
}

\begin{abstract}
Background: Although chemoprophylaxis remains an important strategy for preventing malaria in travellers, its effectiveness may be compromised by lack of adherence. Inappropriate use of chemoprophylaxis is likely to increase the risk of acquiring malaria, but may probably also worsen the severity of imported cases. The aim of this study was to assess the impact of use of malaria chemoprophylaxis on clinical features and outcome of imported malaria.
\end{abstract}

Methods: Demographic, clinical and laboratory data of patients included in the Rotterdam Malaria Cohort between 1998 and 2011 were systematically collected and analysed. Patients were classified as self-reported compliant or non-compliant users or as non-users of chemoprophylaxis. Severe malaria was defined using the 2010 WHO criteria.

Results: Details on chemoprophylaxis were available for 559 of the 604 patients, of which $64.6 \%$ were non-users, $17.9 \%$ were inadequate users and $17.5 \%$ reported to be adequate users. The group of non-users was predominated by patients with African ethnicity, partial immunity and people visiting friends and relatives. The majority contracted Plasmodium falciparum malaria. In contrast, compliant users acquired non-falciparum malaria more frequently, had significant lower $P$. falciparum loads on admission, shorter duration of hospitalization and significant lower odds for severe malaria as compared with non-users. Patients with $P$. falciparum malaria were more likely to have taken their chemoprophylaxis less compliantly than those infected with non-P. falciparum species. Multivariate analysis showed that self-reported adequate prophylaxis and being a partially immune traveller visiting friends and relatives was associated with significantly lower odds ratio of severe malaria. In contrast, age, acquisition of malaria in West-Africa and being a non-immune tourist increased their risk significantly.

Conclusions: Compliant use of malaria chemoprophylaxis was associated with significantly lower odds ratios for severe malaria as compared with non-compliant users and non-users of chemoprophylaxis. After correction for age, gender and immunity, this protective effect of malaria chemoprophylaxis was present only in individuals who adhered compliantly to use of chemoprophylaxis. Patients with $P$. falciparum malaria were more likely to have used their chemoprophylaxis less compliantly than patients with non-P. falciparum malaria who were more likely to have contracted malaria in spite of compliant use of chemoprophylaxis.

Keywords: Malaria, Travellers, Prophylaxis, Plasmodium falciparum, Travel, Outcome, Import, Severe, Atovaquone, Proguanil, Mefloquine, Immunity

\footnotetext{
* Correspondence: p.van.genderen@havenziekenhuis.nl

'Institute for Tropical Diseases, Harbour Hospital, Haringvliet 72, 3011, TG

Rotterdam, The Netherlands

Full list of author information is available at the end of the article
}

\section{Biomed Central}

(c) 2013 Vliegenthart-Jongbloed et al.; licensee BioMed Central Ltd. This is an Open Access article distributed under the terms of the Creative Commons Attribution License (http://creativecommons.org/licenses/by/2.0), which permits unrestricted use, distribution, and reproduction in any medium, provided the original work is properly cited. 


\section{Background}

Malaria is a mosquito-transmitted disease which may cause a wide variety of symptoms, ranging from no or mild symptoms to severe disease and death. The clinical features and outcome measures of malaria are thought to depend on the infecting Plasmodium species, the immune status of the patient, prior use or non-use of chemoprophylaxis, and the timeliness and nature of any treatment administered. Even though the global burden of malaria is largely carried by the world's malariaendemic regions with as many as 500 million cases annually and a death toll of one million children each year, malaria may also be acquired by international travellers from non-endemic areas [1]. Every year between 10,000 and 30,000 of these travellers fall ill with malaria after returning home [2]. Estimates indicate that around 150 returning travellers die each year from imported malaria, usually due to Plasmodium falciparum infection [1].

Malaria can be prevented through a range of barrier measures to prevent mosquito bites and by taking chemoprophylactic drugs. In areas of intense malaria transmission, malaria chemoprophylaxis remains the most important strategy for preventing malaria in travellers [3], but its use may be associated with adverse outcomes and even death [4]. These potentially severe adverse effects may undermine compliant use of malaria chemoprophylaxis in travellers, in particular when considering that these travellers were usually healthy when commencing travel [5-9]. An additional difficulty with malaria chemoprophylaxis is that all drug regimens must be taken meticulously during and for one or more weeks after leaving the malaria-endemic area [10]. The compliant use of malaria chemoprophylaxis therefore requires considerable personal discipline. Unfortunately, a substantial proportion of travellers may discontinue their anti-malarial drugs soon after returning home because continuation of these drugs after travel may feel counterintuitive [11]. Inappropriate use or early discontinuation of chemoprophylaxis is likely to be a key element in malaria acquisition, but might, speculatively, also worsen the severity of imported cases. In the present study the impact of (self-reported) adherence to malaria chemoprophylaxis on clinical features and outcome of imported malaria was compared to those who reported less compliant use or did not use malaria chemoprophylaxis at all.

\section{Methods}

The Harbour Hospital is a 161-bed general hospital located in Rotterdam, The Netherlands. It also comprises the Institute for Tropical Diseases, which serves as a national referral centre. The Rotterdam Malaria Cohort consists of all patients diagnosed with malaria at the Institute for Tropical Diseases in Rotterdam. In the period 1998-2011 the Rotterdam Malaria Cohort comprised 604 cases of imported malaria. All patients' demographic, clinical and laboratory data are systematically collected and stored in an electronic database after deidentification. For the present study, data from patients who entered the Rotterdam Malaria Cohort between June 1998 and December 2011 were used to estimate the impact of use of malaria chemoprophylaxis on clinical and laboratory features of imported malaria as well as on the outcome of imported malaria. The outcome measures of interest were: severity of malaria, time in hospital, admission to intensive care unit (ICU), mechanical ventilation, renal replacement therapy, exchange transfusion and death. All available laboratory data were measured on admission with the use of routine procedures.

The standard procedure to diagnose malaria comprised a quantitative buffy coat (QBC) analysis, a rapid diagnostic test (RDT) for malaria antigens Binax $\mathrm{NOW}^{\oplus}$ Malaria Test (Binax, Inc, Maine, USA), and thick and thin blood smears using freshly collected blood specimens from finger pricks. The malaria rapid test and the QBC analysis were performed according to the manufacturer's instructions. QBC capillaries were examined independently by two technicians by microscopic analysis of two complete rows of the region between the bottom of the capillary and the poly-nuclear leukocyte layer using an Olympus BX-60 fluorescence microscope equipped UV-filter and 50x objective and 12.5x oculars (total magnification 625x). Thick blood smears were stained with Field's stain (Brunschwig Chemie, Amsterdam, The Netherlands) and thin smears were fixed with absolute methanol for three minutes and stained with Diff Quick (Medion Diagnostics, Düdingen, Switzerland). Both staining procedures had been optimized for optimal staining of Plasmodium parasites as well as Schüffner's dots and Maurer's clefts in infected erythrocytes. Thick and thin smears were examined with regular light microscopes at a total magnification of $1,250 x$.

\section{Definitions}

\section{Severe malaria}

Patients were considered as having severe P. falciparum malaria if they met the 2010 World Health Organization (WHO) criteria for severe malaria on admission or during hospitalization [12]. These criteria differ from the preset criteria [13] that were used to define severe malaria in previous studies for the Rotterdam Malaria Cohort [14].

\section{Use of malaria chemoprophylaxis}

Compliant use of malaria chemoprophylaxis was defined as self-reported compliant use of malaria chemoprophylaxis, 
i.e., taken in line with the national guidelines for travellers' health advice regarding malaria chemoprophylaxis (www. lcr.nl). In case the patient did not adhere compliantly to these Dutch guidelines, the use of chemoprophylaxis was classified as non-compliant. Travellers not using any drug for chemoprophylaxis of malaria were labelled as nonusers.

\section{Estimation of immunity to malaria}

The degree of immunity to malaria was estimated as follows [15]. Adult immigrants from a malaria-endemic country living in The Netherlands were considered partially immune, because they had likely been exposed to $P$. falciparum during childhood. Immigrant patients who had been born, raised and living in a malaria-endemic area at the time of diagnosis were presumed semiimmune. However, given the relatively low number of semi-immune persons in the Rotterdam Malaria Cohort [15], they were grouped with partially immune individuals. Tourists from non-endemic regions who travelled to endemic areas were considered non-immune.

\section{Statistical analysis}

Data were entered into an Excel database and reviewed for inconsistencies. IBM SPSS statistics 19 (IBM inc, Chicago, IL, USA) was used for statistical analysis, using Chi-square tests for binary outcomes and Kruskal-Wallis analysis to compare continuous variables. Dunn's posthoc calculations were performed using GraphPad Instat 3.0 in case of a significant Kruskal-Wallis result. Univariate and multivariate logistic regression was used to assess the impact of several variables on the outcome parameter severe malaria. P. falciparum parasite load was not entered as an explanatory variable in the univariate and multivariate analyses of predictors for severe disease, because parasite load is - by definition - one of the defining criteria of severe $P$. falciparum malaria. A $P$-value of less than 0.05 was considered to represent a statistically significant difference.

\section{Ethical approval}

Given its retrospective observational design, ethical approval of this study was not required, according to the Dutch Medical Research Involving Human Subjects Act.

\section{Results}

\section{Clinical and laboratory features of malaria patients on admission}

For 559 of 604 (92.5\%) malaria patients included in the Rotterdam Malaria Cohort, details of prophylaxis use were available. The general characteristics of these 559 patients are shown in Table 1. Three hundred and sixtyone (64.6\%) malaria patients did not use any form of malaria chemoprophylaxis, 100 (17.9\%) used malaria prophylaxis inadequately whereas 98 (17.5\%) patients presented with malaria despite adequate use of malaria chemoprophylaxis. Ethnicity differed significantly between the three prophylaxis groups with Caucasians being over-represented in the adequate prophylaxis group. African ethnicity was more common in the group of patients not using malaria chemoprophylaxis. When immunity status towards malaria was estimated, the group of patients without chemoprophylaxis was predominated by patients with presumed partial immunity. The presumed immune status of the majority of adequate users of malaria chemoprophylaxis was non-immune (Table 1). With regard to purpose of travel, tourists comprised a substantial proportion of the adequate users group, whereas the group of non-users was dominated by visiting friends and relatives (VFRs) and sailors. There were no significant differences in seasons of travel. The majority of the non-users contracted malaria in Africa, whereas almost half of the patients with compliant use of chemoprophylaxis contracted malaria outside of Africa. Vital signs on admission did not differ between the three patient groups. Admission C-reactive protein and creatinine levels were significantly higher in non-users as compared with compliant users (Table 1).

\section{Outcome of malaria patients}

Patients with compliant use of malaria chemoprophylaxis had significantly lower odds ratios for the outcome parameter severe malaria (OR 0.121 (95\% CI 0.029-0.516) than patients not using malaria prophylaxis (Table 2, Figure 1). In addition, patients with compliant use of malaria chemoprophylaxis also had lower odds ratios for admission to ICU and shorter times in hospital (Table 2). Also in univariate and subsequent multivariate analyses, age, region of acquisition, adequate use of malaria chemoprophylaxis as well as non-immune tourists and partially immune VFRs were identified as independent predictors for severe malaria (Table 3). Interestingly, non-immune tourists had significant higher odds ratios whereas partially-immune VFRs had significantly lower odds ratios for severe malaria. The combined variables non-immune tourists and partially immune VFRs were used to diminish the number of variables in the multivariate analyse but also to partially negotiate for the (confounding) interaction between non-immunity and tourists on one hand and partially-immunity and VFRs on the other hand.

\section{Causative Plasmodium species in relation to use of malaria chemoprophylaxis}

The distribution of the causative Plasmodium species in relation to the use of and adherence with malaria chemoprophylaxis is shown in Figure 2. The majority of the Plasmodium species in malaria patients who did not use malaria chemoprophylaxis or did not compliantly adhere 
Table 1 General characteristics of malaria patients entered in the Rotterdam Malaria Cohort grouped according to self-reported adherence with malaria chemoprophylaxis

\begin{tabular}{|c|c|c|c|c|}
\hline & $\begin{array}{l}\text { Non-users } \\
n=361\end{array}$ & $\begin{array}{l}\text { Non-compliant users } \\
n=100\end{array}$ & $\begin{array}{l}\text { Compliant users } \\
\mathrm{n}=98\end{array}$ & P-values \\
\hline \multicolumn{5}{|l|}{ Demographics } \\
\hline Age, years & $39(5-70)$ & $37(13-60)$ & $38(4-77)$ & N.S. \\
\hline Gender, n (\%) & & & & N.S. \\
\hline Male & $263(73)$ & $73(73)$ & $62(63)$ & \\
\hline female & $98(27)$ & $27(27)$ & $36(37)$ & \\
\hline Ethnicity, n (\%) & & & & $<0.001$ \\
\hline Caucasian & $148(42)$ & $64(65)$ & $78(80)$ & \\
\hline African & $160(45)$ & $31(31)$ & $14(14)$ & \\
\hline Asian & $32(22)$ & $3(5)$ & $3(3)$ & \\
\hline Other & $16(10)$ & $1(3)$ & $3(3)$ & \\
\hline Immunity, n (\%) & & & & $<0.001$ \\
\hline Non immune & $138(49)$ & $50(64)$ & $30(81)$ & \\
\hline Partially immune & $139(61)$ & $28(36)$ & $7(19)$ & \\
\hline \multicolumn{5}{|l|}{ Travel history } \\
\hline Reason for travel, n (\%) & & & & $<0.001$ \\
\hline Tourist & $69(21)$ & $28(30)$ & $35(51)$ & \\
\hline VFR & $114(35)$ & $27(29)$ & $11(16)$ & \\
\hline Business & $54(17)$ & $27(29)$ & $15(22)$ & \\
\hline Expat & $25(8)$ & $4(4)$ & $3(4)$ & \\
\hline Sailor & $37(11)$ & $3(3)$ & $4(6)$ & \\
\hline Other & $24(7)$ & $4(4)$ & $1(1)$ & \\
\hline Continent of infection, $\mathrm{n}(\%)$ & & & & $<0.001$ \\
\hline African & $290(82)$ & $82(82)$ & $54(58)$ & \\
\hline Asian & $42(12)$ & $10(10)$ & $22(24)$ & \\
\hline Americas & $19(5)$ & $7(7)$ & $14(15)$ & \\
\hline Other & $1(0)$ & $1(1)$ & $3(3)$ & \\
\hline Duration of complaints, n (\%) & & & & N.S. \\
\hline Less than 8 days & $228(63)$ & $70(70)$ & $46(47)$ & \\
\hline 8-14 days & $66(18)$ & $18(18)$ & $21(21)$ & \\
\hline 15-21 days & $25(7)$ & $6(6)$ & $6(6)$ & \\
\hline$>28$ days & $9(3)$ & $5(5)$ & $2(2)$ & \\
\hline \multirow[t]{2}{*}{ Unknown } & $34(9)$ & $1(1)$ & $23(24)$ & \\
\hline & $n=361$ & $n=100$ & $n=98$ & \\
\hline \multicolumn{5}{|l|}{ Vital signs } \\
\hline Temperature, ${ }^{\circ} \mathrm{Celsius}$ & $38.5(35.0-41.5)$ & $38.8(36.1-40.9)$ & $38.5(36.0-41.2)$ & N.S. \\
\hline Systolic blood pressure, mmHg & $120(64-185)$ & $123(90-190)$ & 120 (90-196) & N.S. \\
\hline Pulse rate, beats per minute & $92(50-150)$ & $94(56-130)$ & $96(60-150)$ & N.S. \\
\hline \multicolumn{5}{|l|}{ Laboratory features } \\
\hline Haemoglobin, mmol/L & $8.3(2.5-11.0)$ & $8.3(4.0-11.1)$ & $8.4(4.2-10.7)$ & N.S. \\
\hline Thrombocytes, $\times 10^{9} / \mathrm{L}$ & $84(2-293)$ & $99(3-302)$ & $96(19-258)$ & N.S. \\
\hline Leucocyte count, $\times 10^{9} / \mathrm{L}$ & $5.3(1.3-18.5)$ & $5.1(1.9-13.4)$ & $5.0(1.5-12.9)$ & N.S. \\
\hline CRP, mg/L & $96(5-476)$ & $77(7-310)$ & $71(6-287)$ & $0.0009^{A: p} p<0.01, B: p<0.05$ \\
\hline
\end{tabular}


Table 1 General characteristics of malaria patients entered in the Rotterdam Malaria Cohort grouped according to self-reported adherence with malaria chemoprophylaxis (Continued)

\begin{tabular}{|c|c|c|c|c|}
\hline Creatinine, $\mu \mathrm{mol} / \mathrm{L}$ & $93(39-1.081)$ & $95(55-213)$ & $87(46-405)$ & $0.0019^{B:} p<0.01, C: P<0.01$ \\
\hline $\mathrm{LDH}, \mathrm{U} / \mathrm{L}$ & 265 (103-2.297) & $270(98-877)$ & $237(98-664)$ & N.S. \\
\hline Bilirubin, $\mu \mathrm{mol} / \mathrm{L}$ & $24(3-416)$ & $22(6-95)$ & $23(5-262)$ & N.S. \\
\hline Lactate, $\mathrm{mmol} / \mathrm{L}$ & $1.6(0.5-6.2)$ & $1.6(0.5-4.7)$ & $1.3(1.1-1.8)$ & N.S. \\
\hline
\end{tabular}

Legend: $n=559$. Data are given as median (range) or number (\%), as appropriate.

A: No prophylaxis versus inadequate prophylaxis, B: no prophylaxis versus adequate prophylaxis, C: inadequate prophylaxis versus adequate prophylaxis,

N.S. not statistically significant.

to malaria chemoprophylaxis were identified as $P$. falciparum. In contrast, non-P. falciparum species were identified as the causative species of malaria in the majority of malaria patients who used malaria chemoprophylaxis compliantly (Figure 3). In fact, of the group of malaria patients who reported use of malaria chemoprophylaxis (either compliantly or non-compliantly), patients with $P$. falciparum malaria had significantly higher odds ratio for non-compliant use than patients who presented with non-P. falciparum malaria (Table 4, Figure 3). At the level of specified drug use, this observation was also valid in atovaquone-proguanil and mefloquine users but

Table 2 Outcome measures of patients with malaria, grouped according to self-reported adherence with malaria chemoprophylaxis

\begin{tabular}{|c|c|c|c|c|}
\hline Outcome measure & Group & $\begin{array}{l}\text { Proportion or } \\
\text { mean }(95 \% \mathrm{Cl})\end{array}$ & Odds ratio $(95 \% \mathrm{Cl})$ & $\begin{array}{l}P \text {-value (with no } \\
\text { chemoprophylaxis } \\
\text { as comparator) }\end{array}$ \\
\hline Severe malaria & No chemoprophylaxis & $53 / 361$ & Reference & \\
\hline \multirow[t]{2}{*}{ (WHO 2010) } & Non-compliant use & $8 / 100$ & $0.505(0.232-1.102)$ & N.S. \\
\hline & Compliant use & $2 / 98$ & $0.121(0.029-0.516)$ & 0.0002 \\
\hline \multirow[t]{3}{*}{ ICU admission } & No chemoprophylaxis & $73 / 361$ & Reference & \\
\hline & Non-compliant use & $15 / 100$ & $0.696(0.380-1.276)$ & N.S. \\
\hline & Compliant use & $6 / 98$ & $0.257(0.108-0.611)$ & 0.0008 \\
\hline \multirow[t]{3}{*}{ Mechanical ventilation } & No chemoprophylaxis & $3 / 361$ & Reference & \\
\hline & Non-compliant use & $1 / 100$ & $1.205(0.124-11.722)$ & N.S. \\
\hline & Compliant use & $0 / 98$ & $0.520(0.027-10.158)$ & N.S. \\
\hline \multirow[t]{3}{*}{ Renal replacement therapy } & No chemoprophylaxis & $7 / 361$ & Reference & \\
\hline & Non-compliant use & $0 / 100$ & $0.235(0.013-4.150)$ & N.S. \\
\hline & Compliant use & $0 / 98$ & $0.240(0.014-4.241)$ & N.S. \\
\hline \multirow[t]{3}{*}{ Exchange transfusion } & No chemoprophylaxis & $35 / 361$ & Reference & \\
\hline & Non-compliant use & $6 / 100$ & $0.595(0.243-1.457)$ & N.S. \\
\hline & Compliant use & $1 / 98$ & $0.096(0.013-0.710)$ & 0.0024 \\
\hline \multirow[t]{3}{*}{ Death } & No chemoprophylaxis & $2 / 361$ & Reference & \\
\hline & Non-compliant use & $0 / 100$ & $0.715(0.034-15.033)$ & N.S. \\
\hline & Compliant use & $0 / 98$ & $0.730(0.035-15.341)$ & N.S. \\
\hline \multirow[t]{3}{*}{ P. falciparum species } & No chemoprophylaxis & $285 / 358^{*}$ & Reference & \\
\hline & Non-compliant use & $77 / 100$ & $0.858(0.504-1.460)$ & N.S. \\
\hline & Compliant use & $41 / 98$ & $0.184(0.114-0.297)$ & $<0.0001$ \\
\hline \multirow{3}{*}{$\begin{array}{l}\text { P. falciparum parasite load } \\
\text { (asexual parasites per } \mu \mathrm{L} \text { ) }\end{array}$} & No chemoprophylaxis & $73,774(55,620-91,928)$ & Reference & \\
\hline & Non-compliant use & $48,866(20,264-77,467)$ & N.A. & N.S. \\
\hline & Compliant use & $24,563(5,163-43,963)$ & N.A. & 0.0004 \\
\hline \multirow[t]{3}{*}{ Time in hospital (days) } & No chemoprophylaxis & $4.8(4.4-5.2)$ & Reference & \\
\hline & Non-compliant use & $4.8(4.4-5.3)$ & N.A. & N.S. \\
\hline & Compliant use & $2.9(2.4-3.5)$ & N.A. & $<0.0001$ \\
\hline
\end{tabular}




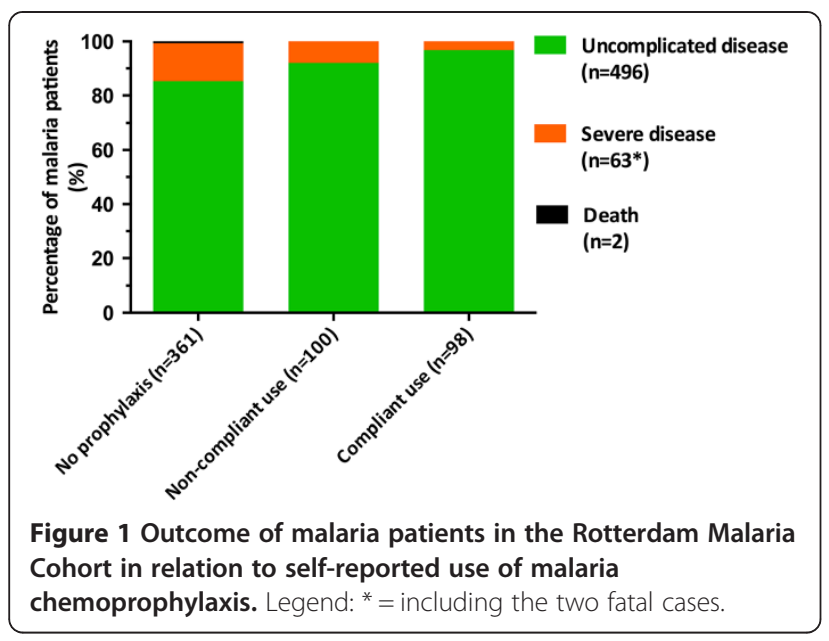

not in chloroquine-proguanil users. Conversely, malaria patients who presented with non- $P$. falciparum species were more likely to have contracted malaria in spite of compliant use of chemoprophylaxis (Table 4, Figure 3).

\section{Causative Plasmodium species in relation to outcome severe malaria}

Even though the occurrence of severe malaria is not explicitly confined to $P$. falciparum species alone and severe cases of non- $P$. falciparum have been recognized (especially in cases with $P$. vivax and $P$. knowlesi infections), all cases of severe malaria in the Rotterdam Malaria Cohort were associated with P. falciparum infections. For this reason, the univariate and multivariate statistical analysis was also done separately for malaria patients with infections solely caused by $P$. falciparum

Table 3 Univariate and multivariate logistic regression of predictors for severe malaria

\begin{tabular}{|c|c|c|c|c|c|}
\hline \multirow[t]{2}{*}{ Variables } & & \multicolumn{2}{|c|}{ Univariate analysis } & \multicolumn{2}{|c|}{ Multivariate analysis } \\
\hline & & $P$-value & Odds ratio $(95 \% \mathrm{Cl})$ & $P$-value & Odds ratio $(95 \% \mathrm{Cl})$ \\
\hline Age & & 0.000 & $1.048(1.024-1.071)$ & 0.003 & $1.037(1.013-1.062)$ \\
\hline \multirow[t]{2}{*}{ Sexe } & Male & 0.256 & $0.726(0.417-1.262)$ & 0.181 & $0.661(0.360-1.213)$ \\
\hline & Female & 0.256 & $1.378(0.792-2.398)$ & & \\
\hline \multirow[t]{2}{*}{ Immunity } & Non-immune & 0.000 & $3.944(2.249-6.915)$ & & \\
\hline & Partially or semi immune & 0.860 & $0.950(0.537-1.681)$ & & \\
\hline \multirow[t]{6}{*}{ Reason for travel } & Tourist & 0.196 & $1.464(0.822-2.608)$ & & \\
\hline & $V F R$ & 0.036 & $0.470(0.233-0.950)$ & & \\
\hline & Business & 0.676 & $1.154(0.590-2.258)$ & & \\
\hline & Expat & 0.175 & $1.903(0.751-4.819)$ & & \\
\hline & Sailor & 0.050 & $2.195(1.001-4.812)$ & & \\
\hline & Other & 0.450 & $0.570(0.132-2.454)$ & & \\
\hline \multirow[t]{6}{*}{ Region of acquisition } & Outside Africa & 0.003 & $0.288(0.128-0.647)$ & & \\
\hline & North Africa & 0.999 & N.A. & & \\
\hline & West Africa & 0.000 & 3.567 (1.969-6.462) & 0.000 & $3.287(1.753-6.161)$ \\
\hline & Central Africa & 0.142 & $0.409(0.124-1.350)$ & & \\
\hline & East Africa & 0.356 & $0.639(0.246-1.656)$ & & \\
\hline & Southern Africa & 0.543 & $1.984(0.218-19.033)$ & & \\
\hline \multirow[t]{2}{*}{ Season of infection } & March-August & 0.362 & $0.780(0.457-1.331)$ & & \\
\hline & September-February & 0.362 & $1.283(0.751-2.190)$ & & \\
\hline \multirow[t]{3}{*}{ Prophylaxis } & Adequate prophylaxis & 0.006 & $0.137(0.033-0.569)$ & 0.007 & $0.132(0.031-0.567)$ \\
\hline & Inadequate prophylaxis & 0.257 & $0,639(0.294-1.387)$ & 0.143 & $0.540(0.236-1.233)$ \\
\hline & No prophylaxis & 0.001 & $3.235(1.607-6.512)$ & & \\
\hline \multirow[t]{4}{*}{ Duration of illness before diagnosis } & 1 to 7 days & 0.670 & $0.882(0.496-1.568)$ & & \\
\hline & 8 to 14 days & 0.115 & $1.633(0.887-3.005)$ & & \\
\hline & 15 to 28 days & 0.478 & $0.644(0.196-2.168)$ & & \\
\hline & More than 28 days & 0.478 & $0.644(0.196-2.168)$ & & \\
\hline \multirow[t]{2}{*}{ Combined variables } & Non-immune tourists & 0.000 & $3.274(1.770-6.058)$ & 0.026 & $2.200(1.101-4.396)$ \\
\hline & Partially-immune VFR & 0.059 & $0.476(0.221-1.029)$ & 0.045 & $0.426(0.185-0.981)$ \\
\hline
\end{tabular}




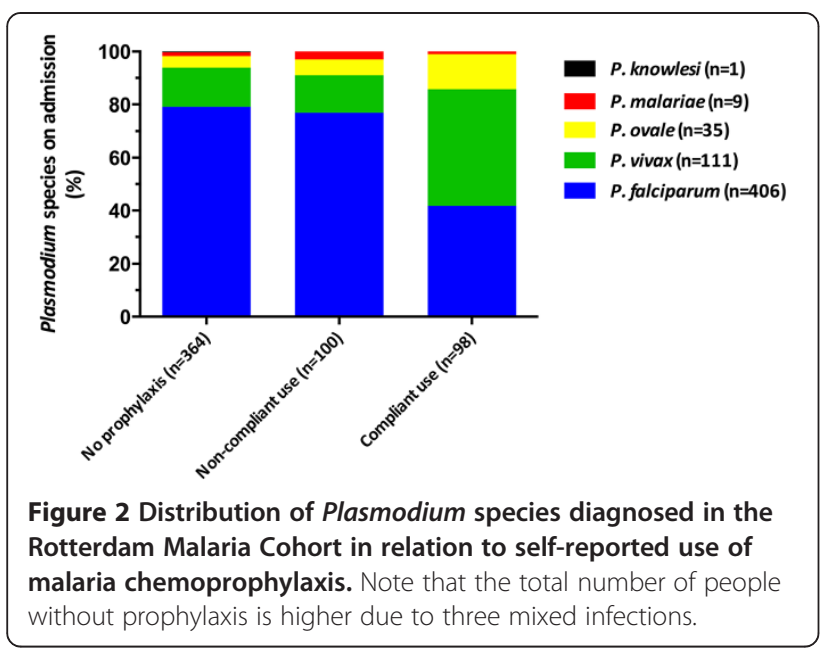

species (Additional file 1). The parameters age, region of acquisition, adequate use of malaria chemoprophylaxis and the combined variable partially-immune VFR were again identified as independent predictors for severe ( $P$. falciparum) malaria but the combined variable non-immune tourists lost its statistical significance due to the reduction in number of eligible cases.

\section{Discussion}

Deaths from malaria among travellers are known to be related to delays in treatment and failure to comply with anti-malarial chemoprophylaxis [16]. Both case fatalities in the current study occurred in patients not taking chemoprophylaxis. However, statistical analysis did not reveal a significant difference in case-fatality rates between compliant users, non-compliant users and non-users, which was conceivably due to the very low number of case fatalities in the Rotterdam Malaria Cohort.
Evidence is accumulating that severe $P$. falciparum malaria tends to occur more commonly in patients not taking malaria chemoprophylaxis [17]. This may be particularly true for the non-immune traveller to malariaendemic regions, especially elderly persons $[18,19]$ as became also evident from the current study. Compliant use of anti-malarial chemoprophylaxis was associated with a significantly lower odds ratio for severe malaria. In addition, compliant users of chemoprophylaxis with malaria had significantly lower P. falciparum loads on admission as compared to those who took no chemoprophylaxis at all or did not comply with proper use. This reduced parasite burden in compliant users of chemoprophylaxis probably directly relates to a decrease in risk since many studies documented that high parasite loads are independently associated with worse outcome $[18,20]$. In terms of outcome, non-compliant users did not significantly differ from non-users. The protective effects of the currently recommended drugs for chemoprophylaxis of malaria was only present when taken as indicated.

Among travellers there is also increasing evidence that severe malaria tends to occur less frequently in people of African origin [21,22]. This reduced risk is commonly attributed to some degree of residual immunity towards malaria. The findings of the present study are in line with this. Individuals with partial immunity visiting friends and relatives had significant lower odds of severe malaria. Conversely, non-immune tourists had significant higher odds for severe malaria and a more complicated course. Even when the protective effects of partial immunity and the risk of non-immunity were taken into account in the multivariate analysis, the protective effect of compliant use of chemoprophylaxis on odds for severe malaria remained present as an independent factor. This suggests that partially immune VFRs may further reduce their risk for severe malaria by strict adherence
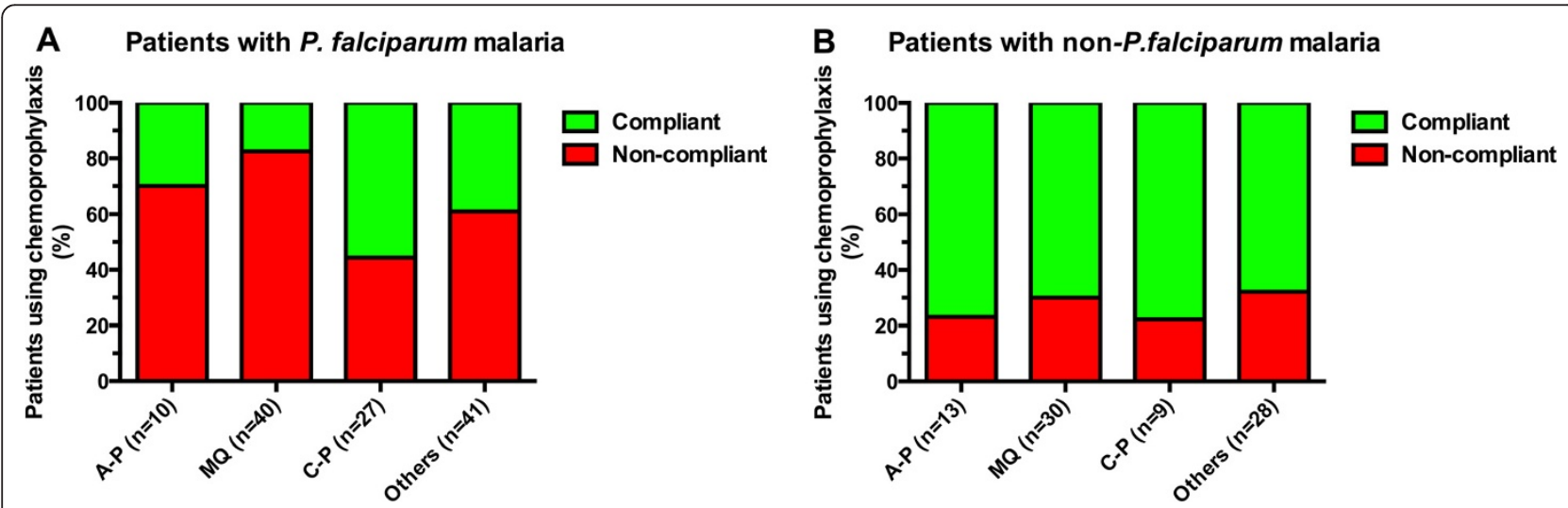

Figure 3 The proportion of compliant versus non-compliant use of specified chemoprophylaxis in patients with malaria. A: in patients presenting with $P$. falciparum malaria, B: in patients presenting with non-P. falciparum malaria. Legend: A-P = atovaquone-proguanil; $\mathrm{MQ}=$ mefloquine; $\mathrm{C}-\mathrm{P}=$ chloroquine-proguanil. 
Table 4 Self-reported adherence with and drugs used for malaria chemoprophylaxis in relation to causative Plasmodium species

\begin{tabular}{|c|c|c|c|c|c|}
\hline \multirow[b]{2}{*}{ Drug used for chemoprophylaxis } & \multirow[b]{2}{*}{ Malaria species } & \multicolumn{2}{|c|}{$\begin{array}{l}\text { Self-reported adherence } \\
\text { to chemoprophylaxis }\end{array}$} & \multirow[b]{2}{*}{$P$-value } & \multirow{2}{*}{$\begin{array}{c}\text { The odds ratio for non-compliant } \\
\text { use of prophylaxis in } P \text {. falciparum } \\
\text { infections versus non-P. falciparum } \\
\text { infections } \\
\text { Odds ratio }(95 \% \mathrm{Cl})\end{array}$} \\
\hline & & Non compliant $(n=100)$ & Compliant $(n=98)$ & & \\
\hline \multirow[t]{2}{*}{ Atovaquone-proguanil } & P. falciparum & 7 & 3 & \multirow{2}{*}{0.024} & \multirow{2}{*}{$7.778(1.200-50.424)$} \\
\hline & Non-falciparum & 3 & 10 & & \\
\hline \multirow[t]{2}{*}{ Mefloquine } & P. falciparum & 33 & 7 & \multirow{2}{*}{$<0.001$} & \multirow{2}{*}{$11.000(3.556-34.023)$} \\
\hline & Non-falciparum & 9 & 21 & & \\
\hline \multirow[t]{2}{*}{ Chloroquine-proguanil* } & P. falciparum & 12 & 15 & \multirow{2}{*}{0.236} & \multirow{2}{*}{$2.800(0.489-16.036)$} \\
\hline & Non-falciparum & 2 & 7 & & \\
\hline \multirow[t]{2}{*}{ Others } & P. falciparum & 25 & 16 & \multirow{2}{*}{0.019} & \multirow{2}{*}{$3.299(1.200-9.069)$} \\
\hline & Non-falciparum & 9 & 19 & & \\
\hline \multirow[t]{2}{*}{ Total } & P. falciparum & 77 & 41 & \multirow{2}{*}{$<0.001$} & \multirow{2}{*}{$4.654(2.517-8.607)$} \\
\hline & Non-falciparum & 23 & 57 & & \\
\hline
\end{tabular}

Legend: N.A. not applicable, $\mathrm{Cl}$ confidence interval, ${ }^{*}=$ chloroquine-proguanil combination only.

to anti-malarial chemoprophylaxis. Of note, and in line with other studies [19], increasing age was also in the current study identified as an independent risk factor for a worse outcome. Pre-travel health advice should stress the importance of compliant use of chemoprophylaxis in each traveller but - given their risk profile [23] - particularly in elderly travellers, non-immune tourists and lastminute-travellers [19,23].

In areas of intense malaria transmission, malaria chemoprophylaxis remains the most important strategy for prevention of malaria in non-immune travellers. A recent Cochrane review, however, provided inconclusive evidence about which of the currently recommended drugs atovaquone/proguanil, mefloquine and doxycycline, was most effective in preventing malaria in non-immune populations travelling to regions with $P$. falciparum resistance to chloroquine [24]. Interestingly, occasional failures of anti-malarial chemoprophylaxis have been documented for any chemoprophylactic regimen [25-27]. The current findings of the acquisition of malaria by travellers despite compliant use of malaria chemoprophylaxis underline these potential restrictions. Even though it can not be excluded that resistance patterns towards the commonly used chemoprophylactic drugs might have changed over time during the course of this study, the findings of the current study stress the notion that the occurrence of $P$. falciparum malaria in a person taking malaria chemoprophylaxis was more likely to be associated with non-compliant than with compliant use. This finding was not only demonstrable in the whole group of malaria patients taking chemoprophylactic drugs, but also valid for those individuals taking either atovaquone/proguanil or mefloquine for prevention of malaria, in line with their biochemical mode of action.
In contrast, this differential effect was not present in chloroquine-proguanil users, which might raise additional concerns on the efficacy of chloroquine-proguanil for prevention of $P$. falciparum malaria. In contrast, the occurrence of non-P. falciparum malaria in a subject taking malaria chemoprophylaxis was more likely to occur in spite of compliant use of a chemoprophylactic drug. This observation is not surprisingly since the labelled indication of chemoprophylactic anti-malarials is to prevent the acquisition of $P$. falciparum malaria only [25-27].

\section{Limitations}

A major limitation of any retrospective analysis is that data were not originally recorded with this type of study in mind. In addition, there is no exact information about the total number of travellers that were at risk of malaria and their compliance with regard to prophylaxis during the study period. This limits the outcomes to odds ratios instead of relative risks. Further, one could argue that estimating the degree of compliance, especially selfreported compliance, may have introduced an important selection and recall bias. Previous studies comparing self-reported compliance with prophylactic antimalarials and measurements of blood levels showed that patients significantly overestimated their degree of compliance [28]. However, even within the framework of these limitations, the parameter self-reported compliance with malaria chemoprophylaxis should be considered a valuable asset for clinical decision making and risk assessment. With the use of the current straightforward clinical criteria for compliant and non-compliant use of malaria chemoprophylaxis, only compliant use of malaria chemoprophylaxis was identified as an independent 
predictor in multivariate analysis, significantly reducing the risk of severe malaria. These protective effects remained present after adjustment for the potentially confounding protective effects of partial immunity and being a VFR traveller. Of note, these protective effects of anti-malarial chemoprophylaxis were not demonstrable for noncompliant users.

\section{Conclusions}

Compliant use of malaria chemoprophylaxis was associated with significantly lower odds ratios for the occurrence of severe malaria and admission to ICU. These protective effects of malaria chemoprophylaxis on outcome of malaria were only present when malaria chemoprophylaxis was taken as indicated. Increasing age, acquisition of malaria in West Africa and being a nonimmune tourist were identified as independent predictors associated with an increased risk of severe malaria. Partially immune individuals, in particular those visiting friends and relatives had significantly lower odds ratios for severe malaria. These important protective effects of compliant use of malaria chemoprophylaxis should be stressed in future pre-travel health encounters with travellers to tropical regions, especially with presumed nonimmune and elderly travellers.

\section{Additional file}

Additional file 1: Univariate and multivariate logistic regression of predictors for severe $P$. falciparum malaria.

\section{Competing interests}

The authors declare that they have no competing interests.

\section{Authors' contributions}

PJJVG made the design of the study, MEvW and PJJVG participated in collecting data and planning analysis and evaluation of data. RK prepared and maintained the Rotterdam Malaria Cohort database. KVJ performed cleaning and extraction of data. KVJ and PJJVG performed statistical analysis and participated in literature review. PJJVG and KVJ drafted the first version of the manuscript. All authors gave input and revised, reviewed and approved the final version of the manuscript.

\section{Acknowledgements}

The Port of Rotterdam is thanked for their unconditional financial grant to study imported, travel-related infectious diseases.

\section{Author details \\ 'Institute for Tropical Diseases, Harbour Hospital, Haringvliet 72, 3011, TG Rotterdam, The Netherlands. ${ }^{2}$ Harbour Hospital and Institute for Tropical Diseases, Laboratory of Parasitology, Haringvliet 72, 3011, TG Rotterdam, The Netherlands. ${ }^{3}$ Department of Medical Microbiology and Infectious Diseases, Erasmus Medical Centre \& Harbour Hospital, Rotterdam, The Netherlands.}

Received: 6 January 2013 Accepted: 24 June 2013

Published: 31 July 2013

\section{References}

1. Wellens TE, Miller LH: Two worlds of malaria. N Engl J Med 2003, 349:1496-1498.
2. WHO: International travel and health. Geneva: World Health Organization; 2008.

3. Croft $A M$, Garner P: Mefloquine for preventing malaria in non-immune adult travellers. Cochrane Database Syst Rev 2000, 4, CD000138.

4. Cook IF: Malaria prevention. Lancet 1986, 2:229.

5. Overbosch D, Schilthuis H, Bienzle U, Behrens RH, Kain KC: Atovaquoneproguanil versus mefloquine for malaria prophylaxis in nonimmune travelers: Results from a randomized, double-blind study. Clin Infect Dis 2001, 33:1015-1021.

6. Croft AM, Whitehouse DP, Cook GC, Beer MD: Safety evaluation of the drugs available to prevent malaria. Expert Opin Drug Saf 2002, 1:19-27.

7. Van Riemsdijk MM, Sturkenboom MC, Pepplinkhuizen L, Stricker BH: Mefloquine increases the risk of serious psychiatric events during travel abroad: a nationwide case-control study in the Netherlands. J Clin Psychiatry 2005, 66:199-204.

8. Moore DA, Grant AD, Armstrong M, Stümpfle R, Behrens RH: Risk factors for malaria in UK travellers. Trans R Soc Trop Med Hyg 2004, 98:55-63.

9. DePetrillo JC, Singer C, Bergagnini IA, Kolakowski P, Edwards B, Smith MA: Assessment of adherence to atovaquone-proguanil prophylaxis in travellers. J Travel Med 2010, 17:217-220.

10. Cobelens FG, Leentvaar-Kuijpers A: Compliance with malaria chemoprophylaxis and preventative measures against mosquito bites among Dutch travellers. Trop Med Int Health 1997, 2:705-713.

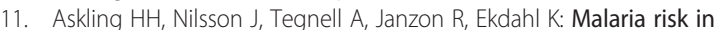
travellers. Emerg Infect Dis 2005, 11:436-441.

12. WHO: Guidelines for the treatment of malaria. Geneva: World Health Organization; 2010. http://www.who.int/entity/malaria/publications/atoz/ 9789241547925/en/

13. Van Genderen PJ, Van der meer IM, Consten J, Petit PL, Van Gool T, Overbosch D: Evaluation of plasma lactate as a parameter for disease severity on admission in travelers with Plasmodium falciparum malaria. J Travel Med 2005, 12:261-264.

14. Van Wolfswinkel ME, Hesselink DA, Zietse R, Hoorn EJ, Van Genderen PJJ: Hyponatraemia in imported malaria is common and associated with disease severity. Malar J 2010, 9:140.

15. Van Genderen PJJ, Hesselink DA, Bezemer JM, Wismans PJ, Overbosch D: Efficacy and safety of exchange transfusion as an adjunct therapy for severe Plasmodium falciparum malaria in nonimmune travellers: a 10-year single-center experience with a standardized treatment protocol. Transfusion 2010, 50:787-794.

16. Christen $D$, Steffen $R$, Schlagenhauf $P$ : Deaths caused by malaria in Switzerland 1988-2002. AmJTrop Med Hyg 2006, 75:1188-1194.

17. Seringe $E$, Thellier M, Fontanet A, Legros F, Bouchaud O: Severe imported Plasmodium falciparum malaria, France, 1996-2003. Emerg Infect Dis 2011, 17:807-813

18. Bruneel F, Tubach F, Corne P, Megarbane B, Mira JP: Severe imported falciparum malaria: a cohort study in 400 critically ill adults. PLoS One 2010, 5:e13236.

19. Checkley AM, Smith A, Smith V, Blaze M, Bradley D, Chiodini PL, Whitty CJM: Risk factors for mortality from imported falciparum malaria in the United Kingdom over 20 years: an observational study. BMJ 2012, 344:e2116.

20. Field JW, Niven JC: A note on prognosis in relation to parasite counts in acute subtertian malaria. Trans R Soc Trop Med Hyg 1937, 30:569-674

21. Lewis SJ, Davidson RN, Ross EJ, Hall AP: Severity of imported falciparum malaria: effect of taking antimalarial prophylaxis. BMJ 1992, 305:741-743.

22. Phillips A, Bassett P, Zeki S, Newman S, Pasvol G: Risk factors for severe disease in adults with falciparum malaria. Clin Infect Dis 2009, 48:871-878

23. Van Genderen PJ, Van Thiel PP, Mulder PG, Overbosch D: Trends in knowledge, attitudes and practices of travel risk groups towards prevention of malaria: results from the Dutch Schiphol Airport Survey 2002 to 2009. Malar J 2012, 11:179.

24. Jacquerioz FA, Croft AM: Drugs for preventing malaria in travellers. Cochrane Database Syst Rev 2009, 4:CD006491.

25. Boggild AK, Parise ME, Lewis LS, Kain KC: Atovaquone-proguanil: report from the CDC expert meeting on malaria chemoprophylaxis (II). Am J Trop Med Hyg 2007, 76:208-223. 
26. Schlagenhauf $P$, Adamcova M, Regep L, Schaerer MT, Rhein HG: The position of mefloquine as a 21st century malaria chemoprophylaxis. Malar J 2010, 9:357.

27. Tan KR, Magill AJ, Parise ME, Arguin PM: Doxycycline for malaria chemoprophylaxis and treatment: report from the CDC expert meeting on malaria chemoprophylaxis. Am J Trop Med Hyg 2011, 84:517-531.

28. Behrens RH, Taylor RB, Pryce DI, Low AS: Chemoprophylaxis compliance in travellers with malaria. J Travel Med 1998, 5:92-94.

doi:10.1186/1475-2875-12-265

Cite this article as: Vliegenthart-Jongbloed et al:: Severity of imported malaria: protective effect of taking malaria chemoprophylaxis. Malaria Journal 2013 12:265.

\section{Submit your next manuscript to BioMed Central and take full advantage of:}

- Convenient online submission

- Thorough peer review

- No space constraints or color figure charges

- Immediate publication on acceptance

- Inclusion in PubMed, CAS, Scopus and Google Scholar

- Research which is freely available for redistribution 